\title{
Ushering a New Era in Gastroenterology: The Flexible Gastroscope
}

\author{
Commentary on: Gastroscopy with a Flexible Gastroscope
}

\author{
Wesley D. Leung • Andres Gelrud
}

Published online: 28 December 2013

(c) Springer Science+Business Media New York 2013

Flexible endoscopy is currently a major diagnostic and therapeutic tool in the management of patients with digestive disorders. The advent of endosocpy is relative recent, with mucosal visualization in the past reliant for the most part on radiography.

The early pioneers of endoscopy faced some formidable challenges. First, the gastrointestinal (GI) tract is not straight, and second, it is dark. Kussmaul is generally credited with attempting the first gastroscopy in 1868 [1]the subject of his experiment having been a professional sword-swallower. The device, which depended on an external source of light, proved impractical. Although unrecognized at the time, Thomas Edison solved the illumination problem in 1878 , but 25 years elapsed before the incandescent lamp was incorporated into endoscopes. In 1881, Mikulicz reports having used a rigid gastroscope, but abandoned its usage as he felt that the procedure was too dangerous. Innovation in design, particularly in Germany, led to continued developments of the rigid gastroscope; nonetheless, the poor visual results obtained did not justify the discomfort from such a rigid instrument and the additional risk of esophageal perforation. As a result, development of the device was largely abandoned in 1911.

The original article Gastroscopy with a flexible gastroscope by Rudolf Schindler published in American Journal of Digestive Diseases and Nutrition, November 1935, Volume 2, Issue 11, pp. 656-663. Springer.

W. D. Leung · A. Gelrud ( $₫)$

Section of Gastroenterology, Department of Medicine, Center for Pancreatic Disorders, Interventional Endoscopy (CERT), University of Chicago, 5758 S Maryland Ave. MC 9028, Chicago, IL 60637, USA

e-mail: agelrud@uchicago.edu

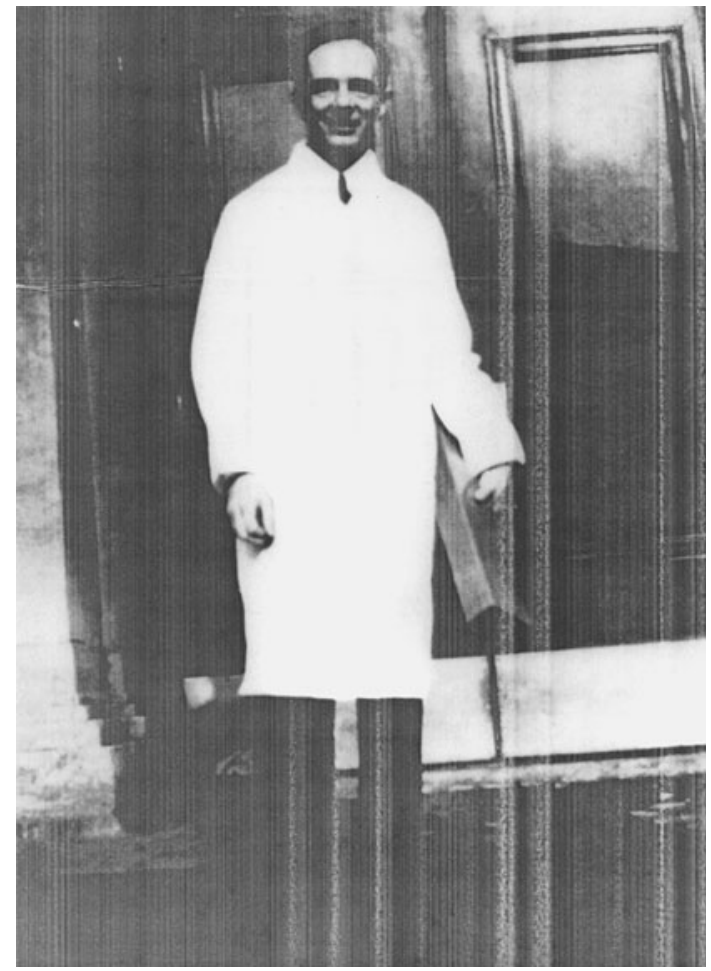

Fig. 1 Rudolph Schindler in front of Billings Hospital (1934) at the University of Chicago

In 1921, Rudolf Schindler at the University of Chicago (Fig. 1) began working again with the rigid gastroscope. He was able to publish his work in an atlas of gastroenterology based on 400 examinations in 1923-the first book containing excellent endoscopic pictures. Figure 2 shows some of the early images obtained by Schindler (as illustrated by Gladys McHugh at the University of Chicago, and provided by Joseph B. Kirsner to David Rubin in 2009). 


\title{
Gastroscopy with a Flexible Gastroscope
}

\author{
By \\ RUDOLF SCHINDLER, M.D. $\dagger$ \\ CHICAGO, ILIINOIS
}

$\mathbf{I}^{\mathrm{T}}$ $\mathrm{T}$ is well known that while gastroscopy has been carried out in an ever-increasing number of cases from 1922 on, the method has not been accepted as a routine procedure in hospitals and clinics or by the gastroenterologists themselves. This has been due, primarily, to the difficulties and the dangers which attended the use of the rigid instrument. In 1932, however, the invention of the flexible gastroscope effected a sudden change whereby the method became at once safe and simple.

Many investigators have used it with success and have published reports. On March 29th, 1934, Benedict in this country reported a series of cases examined with the flexible gastroscope. A few months later Chevalier Jackson, in a paper on gastroscopy, described his experience with this instrument.

Mikulicz first knew, in 1881, that diagnostic gastroscopy could not be made merely with open tubes, but that it had to follow the laws which govern the examination of large cavities in the interior of the body. He saw that gastroscopes would have to be built after the model of cystoscopes, and that the tip of the instrument would have to be equipped with an electric lamp which would radiate its light onto the mucous membrane of the stomach. The reflected rays could be collected by the objective of an optical system, turned by a prism to an angle of $90^{\circ}$ and led to the ocular, through which the examiner was looking. Because of the length of the esophagus, the optical problems of such a system were much more difficult than in cystoscopy. A certain perfection of the optical system was reached by the instruments of LoeningStieda and Elsner (1911). Chevalier Jackson (1907), however, tried to retain the principle of the open esophagoscopical tube. It was easy to show that this was the only possible way for the extraction of foreign bodies through the esophagus. This necessity arises, however, extremely rarely in the practice of the gastroenterologist. The greater need is that of a method which will enable him to diagnose even the smallest lesions of the mucous membrane of the stomach. The open tube is unsatisfactory for this purpose because it is too dangerous and because it does not permit adequate visualization of the interior of the stomach. The difficulties encountered were such that the development of the method was given up in 1911. When I began my work in 1921 there was no longer anyone in the world who used gastroscopy as a diagnostic method.

I used straight, rigid tubes at first. Fig. 1 shows the standard instrument which has been used since

* Presented at the 38th Annual Session of the American Gastro--enterological Association, Atlantic City. N. J., June 10-11, 1935. Approved by the Publications' Committee of 'the Association.
i Visiting Professor of Medicine, University of Chicago. then by many workers. A, is the outer tube with the obturator bearing a rubber finger; $\mathrm{B}$, shows the outer tube after the removal of the obturator and the introduction of the optical tube. With the air balloon, the stomach could be inflated with air. The drawing shows the small holes of the outer tube through which the air passes into the stomach, the mucous membrane of which had to be at least $1 \mathrm{~cm}$. from the objective in order to be seen through the optical system. The astonishing results obtained with this instrument were published in 1923 in a text book and atlas of gastroscopy, based on 400 examinations.

It was nevertheless rather soon apparent that this instrument never could be routinely used. There were two decisive objections. First. the introduction of a rigid tube was never without danger. It was found, in fact, that the introduction of open tubes was even more dangerous than that of closed tubes with rubber obturators. Rupture of the esophagus was possible. The second objection was that, in $10^{\prime} ;$ of all cases, it was not possible to pass through the diaphragmatic portion of the esophagus. A further difficulty was the fact that, in $40 \%$ to $50 \%$ of all cases, it was not possible to introduce the instrument into the lower depths of the stomach, and hence three very important portions of the stomach, the "angle," antrum and pylorus could not be observed.

It was evident that in order to overcome these difficulties it was necessary to build an instrument, the lower part of which, from about $3 \mathrm{~cm}$. above the cardia up to the distal end of the tube, remained flexible during the examination. This instrument had to be capable of being bent in several planes with an angle of at least $30^{\circ}$. This seemed to be impossible. Nevertheless, in cooperation with Wolf of Berlin, we succeeded finally in the construction of such a tube.

Fig. 2 shows this instrument. Its distal end can be bent. The picture seen through the objective is quite sharp and clear unless the tube is bent to an angle of $34^{\circ}$ or more. The rubber finger (A) leads the instrument.* The lamp (B) and the objective (C) are constructed in the usual manner. (D) is the flexible part, which is covered by two thin rubber tubes, between which air can be injected into the stomach. In the newest model the rigid part (E) has a diameter of only $8.5 \mathrm{~mm}$. (F) is the ocular. The optical system (Fig. 3) contains twenty-six lenses and is naturally extremely complicated. For this reason the instrument must be handled very carefully. Danger in this method does not concern the patient, but only the instrument.

The experience of an extended private practice has shown me that gastroscopy can become without any difficulty a routine method of the gastroenterologist,

\footnotetext{
\#A rubber sponge as used hy Henning is unnecessary and not safe.
} 
Fig. 2 Early gastroscopy images. a Gastric ulcer. b Bleeding ulcer. c Distended gastric varices. d Large gastric ulcer eroding into the pancreas. Artwork by Gladys McHugh, the University of Chicago Hospitals, approximately 1940

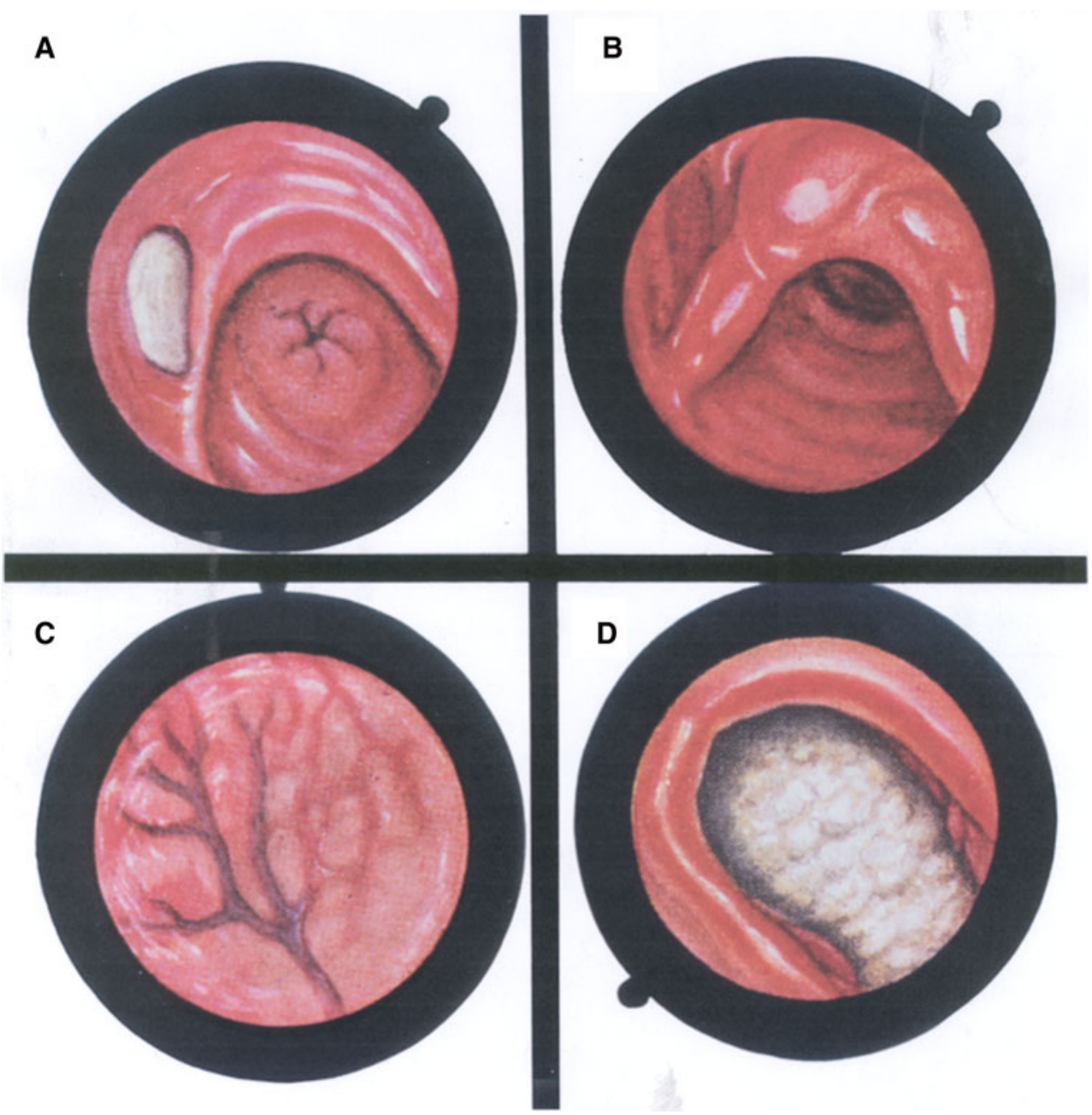

The accompanying article written in 1935 in this journal by Schindler gives the reader a glimpse of how he contributed to the field of gastroenterology and foresaw the future, and represents an example of an innovator in the history of endoscopy from whom we can all learn. Schindler knew that the rigid gastroscope "never could be routinely used" [2] and sought to design an instrument with a flexible lower part from $3 \mathrm{~cm}$ above the cardia to the distal end and which could rotate at least $30^{\circ}$. In collaboration with a fabricator of medical instruments in Berlin, Germany, Schindler was able to design such a device-the flexible gastroscope (Fig. 3).

Schindler's contribution to our knowledge of the normal stomach was monumental at the time and surprisingly accurate. In his article, he describes the stomach as "uniformly orange red in color ... and folds of the mucosa ... crossing and net-like" [2]. He was also able to describe various pathologies of the stomach such as distinguishing between benign and malignant gastric ulcers and proposing a classification of chronic gastritis. Many of his observations at that time were consistent with the conventional beliefs today. and ushered a new era in gastroenterology of morphologic diagnosis that did not depend on X-ray. Of course. with any new technology, there were critics who were slow to adopt this new technology. In the article by Schindler, a physician from Philadelphia, Chevalier Jackson, remarks in response to Schindler's article that many patients "ought to have an open tube [rigid] gastroscopy before he has the flexible gastroscope passed [to rule out esophageal pathology]" [2].

Two important landmarks in the endoscopy revolution occurred later. First, due to the Japanese preoccupation with gastric cancer, doctors at the University of Tokyo and Olympus Optical in 1950 developed the ability to photograph the GI tract (the "gastrocamera"). Second, the first fiber optic endoscope was invented by Basil Hirschowitz and Larry Curtiss in 1957 [3], allowing further exploration of part of the small intestine and the whole colon. From 1968 to 1990, there was an explosion in technical achievements that transformed the practice of gastroenterology. An example of a major breakthrough was the capability to see beyond the mucosa with endoscopic 
Fig. 3 Cartoon of the WolfSchindler gastroscope

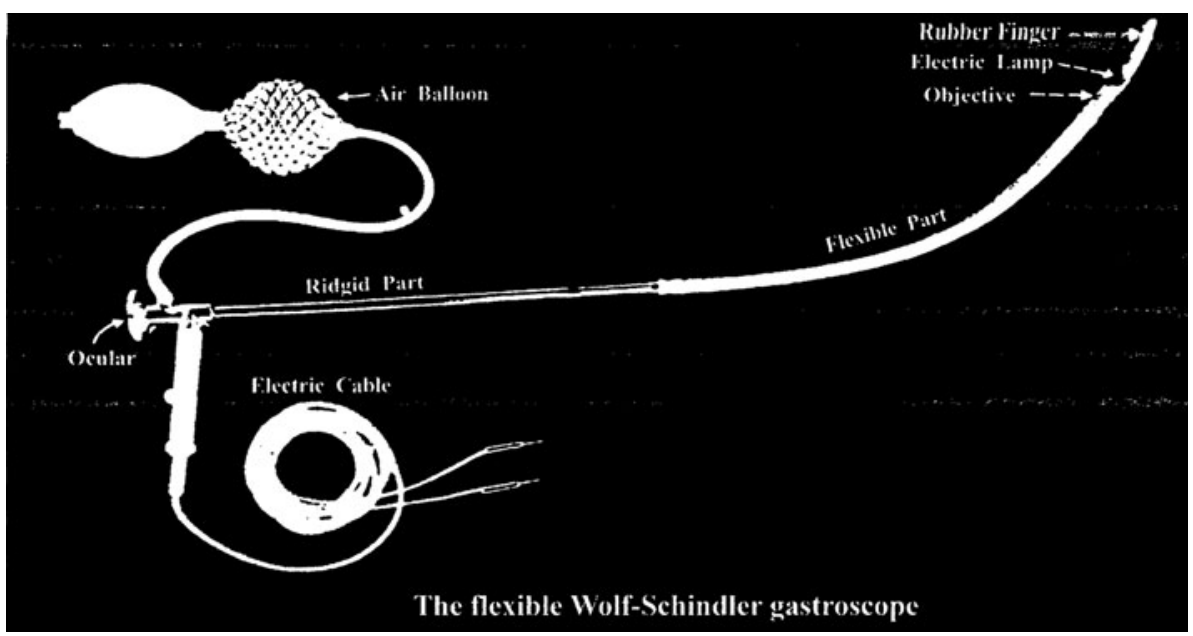

ultrasound. This period is considered by some as the "golden era" of gastrointestinal endoscopy.

Diagnostic endoscopy achieved its current form in 1990, with the widespread adoption of the video-endoscope. The practice of therapeutic endoscopy has evolved since then, however, with the use of endoluminal and biliary stents, variceal band ligation, and endoscopic ultrasound, to name a few, becoming routine. To predict further innovations, it may be helpful to examine the past through articles such as this one by Schindler, from which it is evident that innovation arose from close collaborations between physicians and engineers such as Schindler, and through incorporation of pre-existing technology in fields apart from endoscopy, such as the incandescent light bulb, which took years to be incorporated into the endoscope. Therapeutic endoscopy, for example, might benefit from technologies gained from minimally invasive surgery, interventional radiology, and pathology.

Today, many barriers exist to endoscopic innovation. In the United States, an aging population has busied endoscopists with low-level procedures such as screening colonoscopy. The Medicare reimbursement system favors high-volume procedures, to the detriment of those that are labor-intensive, difficult, and time-consuming. Another factor limiting innovation is scarcity of funding for endoscopic research. To deal with some of these obstacles, more gastroenterologists should be trained to conduct highquality, innovative research, in the mode of Schindler. Moreover, further emphasis should be placed on forging links with engineers and industry. Finally, political pressure should be applied to obtain more funding for endoscopic research, and so be able to continue to provide the best care for our patients. Those who control the funding sources should be reminded that, through endoscopic innovations such as the stent, retrograde cholangioscopy, hemostatic procedures, and venous ligation, to name a few, the need for dangerous, morbid, and costly surgeries has been reduced or eliminated, thus decreasing healthcare costs while improving outcomes. Technical advancements will continue this trend, to the benefit of society.

\section{References}

1. Kluge F, Seidler E. Zur Erstanwendung der Ösophago-und Gastroskopie: Briefe von Adolf Kussmaul und seinen Mitarbeitern. Med Hist J. 1986;21:288-307.

2. Schindler R. Gastroscopy with a flexible gastroscope. Am J Dig Dis Nutr. 1935;2:656-663.

3. Hirshowitz BI, Curtiss LE, Peters CW, Pollard HM. Demonstrations of a new gastroscope, the "fiberscope". Gastroenterology. 1958; 35:50-53. 